OPEN ACCESS

Edited by:

Rena $L i$,

The Roskamp Institute, United States

Reviewed by:

Xingshun $X U$,

Second Affiliated Hospital of

Soochow University, China

Hoa Huu Phuc Nguyen,

University of Tübingen, Germany

*Correspondence:

Baoming $\mathrm{Li}$

bmli@ncu.edu.cn

Hong A. Xu

xuhong@ncu.edu.cn

Xiao-Jiang $\mathrm{Li}$

xli2@emory.edu

Received: 08 January 2019

Accepted: 18 February 2019

Published: 21 March 2019

Citation:

Zhu L, Chen L, Yan L, Perkins BD, Li S, Li B, XU HA and Li X-J (2019)

Mutant Ahi1 Affects Retinal Axon

Projection in Zebrafish via Toxic

Gain of Function.

Front. Cell. Neurosci. 13:81.

doi: 10.3389/fncel.2019.00081

\section{Mutant Ahi1 Affects Retinal Axon Projection in Zebrafish via Toxic Gain of Function}

\author{
Louyin Zhu ${ }^{1,2,3}$, Laiqiang Chen ${ }^{1,4}$, Lingya Yan ${ }^{1}$, Brian D. Perkins ${ }^{5}$, Shihua Li ${ }^{3}$, \\ Baoming $\mathrm{Li}^{1,2 *}$, Hong A. $\mathrm{Xu}^{1,2 *}$ and Xiao-Jiang $\mathrm{Li}^{3 *}$
}

'School of Life Sciences and Institute of Life Science, Nanchang University, Nanchang, China, 2 Jiangxi Provincial Collaborative Innovation Center for Cardiovascular, Digestive and Neuropsychiatric Diseases, Nanchang, China, ${ }^{3}$ Department of Human Genetics, Emory University School of Medicine, Atlanta, GA, United States, ${ }^{4}$ Guangdong-Hongkong-Macau Institute of CNS Regeneration (GHMICR), Jinan University, Guangzhou, China, ${ }^{5}$ Department of Molecular Medicine, Cleveland Clinic Lerner College of Medicine of Case Western Reserve University, Cleveland, OH, United States

Joubert syndrome (JBTS) is an inherited autosomal recessive disorder associated with cerebellum and brainstem malformation and can be caused by mutations in the Abelson helper integration site-1 (AHI) gene. Although AHI1 mutations in humans cause abnormal cerebellar development and impaired axonal decussation in JBTS, these phenotypes are not robust or are absent in various mouse models with Ahi1 mutations. AHI1 contains an N-terminal coiled-coil domain, multiple WD40 repeats, and a C-terminal Src homology $3(\mathrm{SH} 3)$ domain, suggesting that $\mathrm{AH} 1$ functions as a signaling or scaffolding protein. Since most AHI1 mutations in humans can result in truncated AHI1 proteins lacking WD40 repeats and the SH3 domain, it remains unclear whether mutant AHI1 elicits toxicity via a gain-of-function mechanism by the truncated AHI1. Because Ahi1 in zebrafish and humans share a similar N-terminal region with a coiled-coil domain that is absent in mouse Ahi1, we used zebrafish as a model to investigate whether Ahi1 mutations could affect axonal decussation. Using in situ hybridization, we found that ahi1 is highly expressed in zebrafish ocular tissues, especially in retina, allowing us to examine its effect on retinal ganglion cell (RGC) projection and eye morphology. We injected a morpholino to zebrafish embryos, which can generate mutant Ahi1 lacking the intact WD40 repeats, and found RGC axon misprojection and ocular dysplasia in $4 \mathrm{dpf}$ (days post-fertilization) larvae after the injection. However, ahi1 null zebrafish showed normal RGC axon projection and ocular morphology. We then used CRISPR/Cas9 to generate truncated ahi1 and also found similar defects in the RGC axon projection as seen in those injected with ahi1 morpholino. Thus, the aberrant retinal axon projection in zebrafish is caused by the presence of mutant ahi1 rather than the loss of ahi1, suggesting that mutant Ahi1 may affect axonal decussation via toxic gain of function.

Keywords: axonal decussation, AHI1, CRISPR/Cas9, coiled-coil, gain of function 


\section{INTRODUCTION}

Joubert syndrome (JBTS) is a developmental disorder characterized by cerebellar vermis hypoplasia and a midbrainhindbrain malformation, called the molar tooth sign (Joubert et al., 1969; Louie and Gleeson, 2005). JBTS can be caused by mutations in more than 30 genes, and most of these gene products are important for cilia function. The Abelson helper integration site-1 (AHI1) locus was initially identified as a common helper provirus integration site for murine leukemias and lymphomas (Poirier et al., 1988). Later studies revealed that nonsense or frame-shift mutations in AHI1 are associated with JBTS (Dixon-Salazar et al., 2004; Ferland et al., 2004). Genetic mapping and association studies have also identified AHI1 as a susceptibility gene for schizophrenia and autism (Levi et al., 2005; Amann-Zalcenstein et al., 2006; Ingason et al., 2007, 2010; Torri et al., 2010). All these findings suggest that Ahil is important for early brain development and that its dysfunction is involved in neurological and psychiatric disorders that are closely related to abnormal early development.

Mouse models with Ahil mutations have provided very valuable information about the function of Ahi1. Several Ahi1 knockout (KO) mouse models were generated by deletion of exon 2 (Xu et al., 2010), exons 2-5 (Hsiao et al., 2009), or exons 6-7 (Louie et al., 2010). These various mutant mice revealed that Ahil is important for early development and neuronal differentiation, as loss of Ahil can lead to a smaller cerebellum with an underdeveloped vermis (Lancaster et al., 2011), as well as failure of photoreceptor outer segment formation (Louie et al., 2010; Westfall et al., 2010). However, abnormal axonal decussation, a pathological feature of humans with JBTS (Friede and Boltshauser, 1978; Yachnis and Rorke, 1999), was not seen in Ahil mutant mice (Hsiao et al., 2009; Xu et al., 2010; Lancaster et al., 2011).

The lack of abnormal axonal decussation in Ahil mutant mice could be accounted for by differences in Ahil's gene structures and expression in mice vs. humans. Indeed, the $\mathrm{N}$-terminal region of mouse Ahil lacks the coiled-coil domain, which is present in N-terminal Ahil in humans and zebrafish. Because over $80 \%$ of Ahil mutations in humans can yield truncated Ahil containing the N-terminal region but lacking the intact $\mathrm{W} 40$ repeats and $\mathrm{SH} 3$ domain, it is possible that abnormal axonal decussation is caused by the effects of truncated human Ahil on axonal projection. In support of this idea, homozygous nonsense mutations of Ahil in four families did not result in the JBTS phenotypes (Elsayed et al., 2015). Because of similarities in the N-terminal regions of human and fish Ahil proteins, we used zebrafish to examine whether Ahil mutations can affect retinal axon projection. Our findings support the notion that mutant Ahi1 lacking the intact WD40 repeats is able to affect axonal projection in the ocular tissues of zebrafish. Our findings also suggest that in human brains, AHI1 mutations may affect axonal decussation via the toxic gain of function by N-terminal AHI1.

\section{MATERIALS AND METHODS}

\section{Zebrafish Husbandry}

Wild-type $\mathrm{AB}$ zebrafish (Danio rerio) were maintained and raised at an aquatic habitat recirculating water facility on a 14-10 light-dark cycle. Embryos were hatched in embryo medium E3 solution $(5 \mathrm{mM} \mathrm{NaCl}, 0.17 \mathrm{mM} \mathrm{KCl}, 0.33 \mathrm{mM}$ $\mathrm{CaCl}_{2}$, and $0.33 \mathrm{mM} \mathrm{MgSO}_{4}$ ) and kept at $28.5^{\circ} \mathrm{C}$. For suppressing pigmentation, $0.003 \%$ PTU (1-phenyl-2-thiourea, Sigma-Aldrich, St. Louis, MO, USA) was added into E3 solution when necessary. Embryos were staged according to somite numbers or hours post-fertilization (hpf). The ethical review committee of Nanchang University approved all experimental procedures. For investigating axonal projection in Ahi1 KO zebrafish, we used the PFA-fixed $4 \mathrm{dpf}$ ahilri ${ }^{46-/-}$ embryos, which were provided by Dr. Brian Perkins and described previously (Lessieur et al., 2017).

\section{In situ Hybridization}

cDNA probe for Ahil mRNA (exons 3-4) in situ hybridization was generated using the following PCR primers: forward $\left(5^{\prime}-\mathrm{AG}\right.$ TCTCAGGAAATTATCGTGCTT-3') and reverse (5'-TTTTC CTCTTCCCGCTGGTC- $\left.3^{\prime}\right)$. PCR products were purified using a kit (Axygen, AP-PCR-250) and then subcloned into T3 vector (Peasy-T3 Cloning Kit, Transgene). After confirming the DNA sequences by Sangon Biotech, Shanghai, China, the plasmid was digested with NcoI (Takara, 1160A) or SpeI (Takara, 1086A) to linearize cDNA, and then used with an SP6 or a T7 transcription kit to generate DIG-labeled (DIG RNA Labeling Mixture, Roche) anti-sense RNA probes. After purification with a RNA Probe Purification Kit (Omega Bio-Tek, R6248), the probe was diluted with hybridization buffer and stored at $-20^{\circ} \mathrm{C}$ before using.

\section{Whole-Mount In situ Hybridization}

The experiment of whole-mount in situ hybridization was performed as described by Thisse and Thisse (2008). Fish embryos at different developmental stages were fixed in $4 \%$ paraformaldehyde/PBS at $4^{\circ} \mathrm{C}$ overnight, and then dehydrated in methanol with a gradient from 25 to $100 \%$ for storage. When the fixed embryos were analyzed, they were gradiently rehydrated to $0.1 \%$ PBST $(0.1 \%$ Tween- 20 in phosphatebuffered saline). Embryos at different developmental stages (24 hpf to $72 \mathrm{hpf}$ ) were digested with proteinase $\mathrm{K}$ from 1 $\mu \mathrm{g} / \mathrm{ml}$ to $80 \mu \mathrm{g} / \mu \mathrm{l}$ for $6-30 \mathrm{~min}$ based on hpf. The treated embryos were then incubated in digoxin-labeled probes for hybridization at $70^{\circ} \mathrm{C}$ overnight. The embryos were transferred to the preheated 50\% SSCT-50\% hybridization buffer for $30 \mathrm{~min}$ at $70^{\circ} \mathrm{C}$, blocked in $10 \%$ sheep serum in $0.1 \%$ PBST, then incubated with anti-DIG antibody at $4^{\circ} \mathrm{C}$ overnight. The embryos were then incubated in NBT/BCIP (Sangon Biotech, Shanghai, China) staining solution for visualizing stained signals. Embryos were mounted in methylcellulose and were scanned using a Nikon AZ100 microscope with Nikon Digital Sight DS-Fill digital camera (Nikon, Japan). Images were captured and processed with NIS-Elements F 3.0 (Nikon). 


\section{Morpholino Injection}

All morpholinos (MOs) used in this article were obtained from Gene Tools LLC (Philomath, OR, USA). We injected a previously reported ahil splice-blocking morpholino, 5'-CC ACACTCTGAAAGGGAAAAACATT-3' (Simms et al., 2012), which is able to target the junction region between intron 12 and exon 13 of zebrafish ahil (ENSDART00000148403.2). The control MO was 5'-CCTCTTACCTCAGTTACAA TTTATA-3'. Zebrafish embryo yolks (1-2-cell stage) were microinjected with $\mathrm{MO}$ in 1-1.5 $\mathrm{nl}$ of distilled water and $0.5 \%$ phenol red.

\section{PCR and DNA Sequencing}

For testing morpholino targeting efficiency, we collected $30 \mathrm{WT}$ and ahil MO-injected embryos at $3 \mathrm{dpf}$ and extracted their mRNAs for generating cDNA using Reverse Transcriptase M-MLV (RNase H-; 2641Q, Takara). The knockdown efficiency and specificity of ahil were evaluated by PCR with the primers (forward: $5^{\prime}$-AGATGGGCTGTTTTACT CTC-3'; reverse: 5'-TTCCGCAAGGAGTGAACGTA-3'). The intact ahil and truncated PCR products were separated by gel electrophoresis, and the truncated ahil DNA was amplified by AxyPrep DNA Gel Extraction Kit (AP-GX-250G, Axygen) for sequencing (Sangon Biotech, Shanghai, China).

\section{CRISPR/Cas9 Targeting}

The two sites in the fish ahil gene were selected for CRISPR-Cas9 targeting using Benchling ${ }^{1}$. The first target site is located in the region between 19460-19482 of exon 14 and the second target site in the region between 15102-15124 of exon 11 (ENSDART00000148403). We obtained Cas9 protein and crRNA from Integrated DNA Technologies, Inc. USA (IDT) and used the Alt-R CRISPR-Cas9 System (IDT) to synthesize ahi1 gRNAs. The gRNAs and Cas9 enzyme were mixed at the final concentration of $20 \mathrm{ng} / \mathrm{nl}$ and $200 \mathrm{ng} / \mathrm{nl}$ in Nuclease-Free IDTE buffer before injection. The CRISPR/Cas9 gRNA mixture (1-1.5 $\mathrm{nl})$ was injected into embryos at the one-cell stage using a microinjection system (PICOSPRITZER III, Parker, IN, USA). After injection, the injected embryos were collected in E3 solution and incubated at $28.5^{\circ} \mathrm{C}$ for their development.

We collected uninjected WT and the injected embryos at $3 \mathrm{dpf}$ and extracted the genomic DNA for T7EI mismatch assay using a kit (M0302L, NEB). Two pairs of primers (P2: F, 5'-AAA ACC AGC ACT CAG ATA CAG G-3', R, 5'-ACT TTA ACG CAT AAC CAT CG-3'; P6: F, 5'-CAT AAA ACT CTT CCG CAC CCT-3', R, 5'-AGA AGA ATT TTC AAG GCA AGC-3') were used to amplify the target region. The WT and injected embryo genomic DNAs were amplified using Mastercyler X50i (Eppendorf, USA) with $50 \mu \mathrm{l}$ PCR reaction. These two PCR products were mixed, and $\mathrm{T} 7$ endonuclease I was added into the mixture for incubation at $37^{\circ} \mathrm{C}$ for $15 \mathrm{~min}$. The mixture was then analyzed by gel electrophoresis.

The PCR-amplified products were also isolated by gel electrophoresis and extracted using an E.Z.N.A. ${ }^{\circledR}$ Gel Extraction

\footnotetext{
${ }^{1}$ https://benchling.com/academic
}

Kit (D2500901, Omega Bio-Tek, USA). The PCR products were subcloned into T3 vector (Invitrogen ${ }^{\mathrm{TM}} \mathrm{TOPO}^{\mathrm{TM}}$ TA Cloning ${ }^{\mathrm{TM}}$ Kit, 450641, Invitrogen, USA) for DNA sequencing (Macrogen Corp., Rockville, MD, USA).

\section{Retinal Axon Labeling}

Lipophilic dyes were injected separately into the eyes of zebrafish embryos at $4 \mathrm{dpf}$ using a microinjection system with the following dyes: DiI (1,1'-Dioctadecyl3,3,3',3'-Tetramethylindocarbocyanine Perchlorate; Molecular Probes, cat.-no. D-282) and DiD (1,1'-Dioctadecyl-3,3,3',3'Tetramethylindodicarbocyanine Perchlorate; cat. no. D-307). The injected embryos were then incubated at $4^{\circ} \mathrm{C}$ overnight to allow the dyes to travel along the lipid membrane of retinal axons. Imaging analysis was done using a confocal microscope (Olympus FV1000, Japan) with Z-stack model to ensure that a maximum of axon projections were completely scanned. The length of each retinal axon was calculated from the retina structure edge to the end of the retinal tract, and the eye size was measured by threshold model of Image J.

\section{Statistical Analysis}

Results are presented as means \pm SEM, which were obtained using GraphPad Prism 7 (GraphPad Software, San Diego, CA, USA). Unpaired Student's $t$-test was used to compare two groups, and one-way ANOVA with a Tukey's multiple comparison test was used for multiple group comparison. A significant level was considered with $p$ values $<0.05$.

\section{RESULTS}

\section{Ahi1 Structure and Expression in Zebrafish}

Human AHI1 is a cytoplasmic protein containing an $\mathrm{N}$-terminal coiled-coil domain, multiple WD40 repeats, and a C-terminal SH3 domain (Jiang et al., 2002). Its multiple protein-binding domain motifs suggest that AHI1 functions as a signaling or scaffolding protein involved in protein-protein interactions (Jiang et al., 2002; Ferland et al., 2004). AHI1 orthologs are found in all vertebrates and share similar structures of WD40 repeats and the C-terminal SH3 domain (Figure 1A); however, Ahil in mouse and Xenopus lacks the N-terminal coiled-coil that is present in the primate, pig, and zebrafish (Figure 1A). Using the automated protein structure tool ITASSER $^{2}$ to analyze three-dimensional structures of human and fish Ahil, we found that the predicted structures of both human and fish Ahil proteins are highly similar (Figure 1B). On the other hand, mouse Ahil, which lacks an N-terminal coiled-coil domain, shows a three-dimensional structure that is quite different from human and fish Ahil. These differences suggest that the $\mathrm{N}$-terminal coiled-coil domain may influence the structure and interactions of N-terminal AHI1 with other proteins.

It has been reported that ahil expression in fish is also different from expression in mouse, as mouse Ahil is absent in glia and the cerebellum, whereas fish Ahil is ubiquitously

\footnotetext{
$\overline{{ }^{2} \text { https://zhanglab.ccmb.med.umich.edu/I-TASSER/ }}$
} 


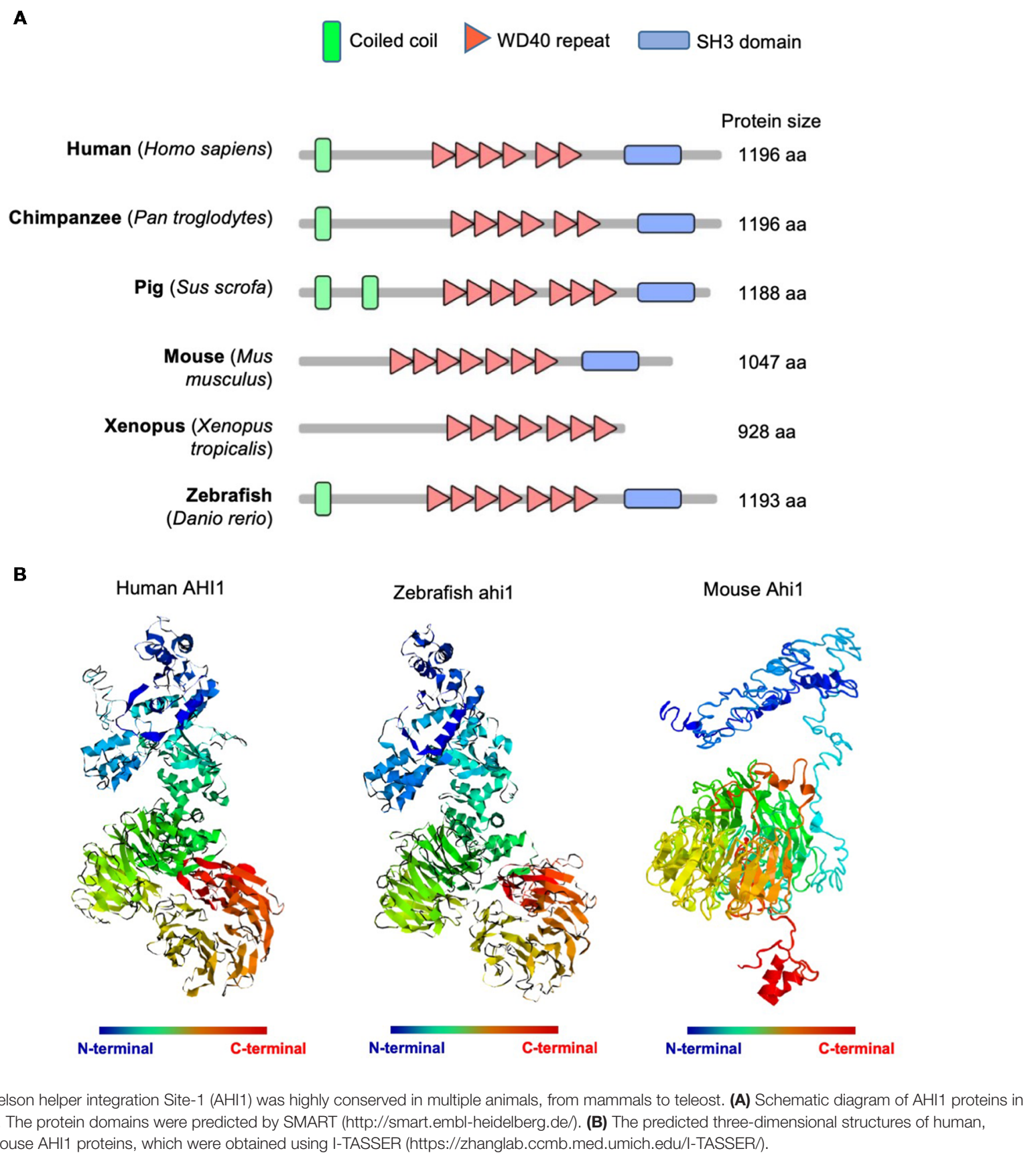

FIGURE 1 | Abelson helper integration Site-1 (AHI) was highly conserved in multiple animals, from mammals to teleost. (A) Schematic diagram of AHI1 proteins in different species. The protein domains were predicted by SMART (http://smart.embl-heidelberg.de/). (B) The predicted three-dimensional structures of human, zebrafish, and mouse AHI1 proteins, which were obtained using I-TASSER (https://zhanglab.ccmb.med.umich.edu/I-TASSER/).

expressed in the whole body from $2.5 \mathrm{dpf}$ to $5.2 \mathrm{dpf}$ (Doering et al., 2008). However, how ahil is expressed in the early developmental stages in zebrafish and whether mutations or loss of ahil affects axonal projection during early brain development remain to be investigated. We therefore performed in situ hybridization using an antisense oligonucleotide probe to exons 3 and 4 in the fish ahil gene. Whole-mount in situ hybridization for ahil mRNA in zebrafish during the early embryonic development stage (from $24 \mathrm{hpf}$ to $72 \mathrm{hpf}$ ) demonstrated that ahi1 is expressed throughout the brain and body (Figure 2A). Importantly, ahi1 expression in the ocular tissues is abundant at very early developmental stages ( $24 \mathrm{hpf}$ and $36 \mathrm{hpf}$ ) and becomes attenuated at $72 \mathrm{hpf}$ (Figure 2B).

\section{Ahi1 Expression and Axonal Projection in Ocular Tissues}

Axonal decussations reflect the midline crossing of nerve tracts from one hemisphere of the brain to the contralateral sense organ or limb. In zebrafish embryos, most retinal ganglion cell (RGC) axons project to the contralateral optic tectum. Due to the simplicity and accessibility of the projection of RGC axons to the contralateral optic tectum in zebrafish, this axonal projection 


\section{A}
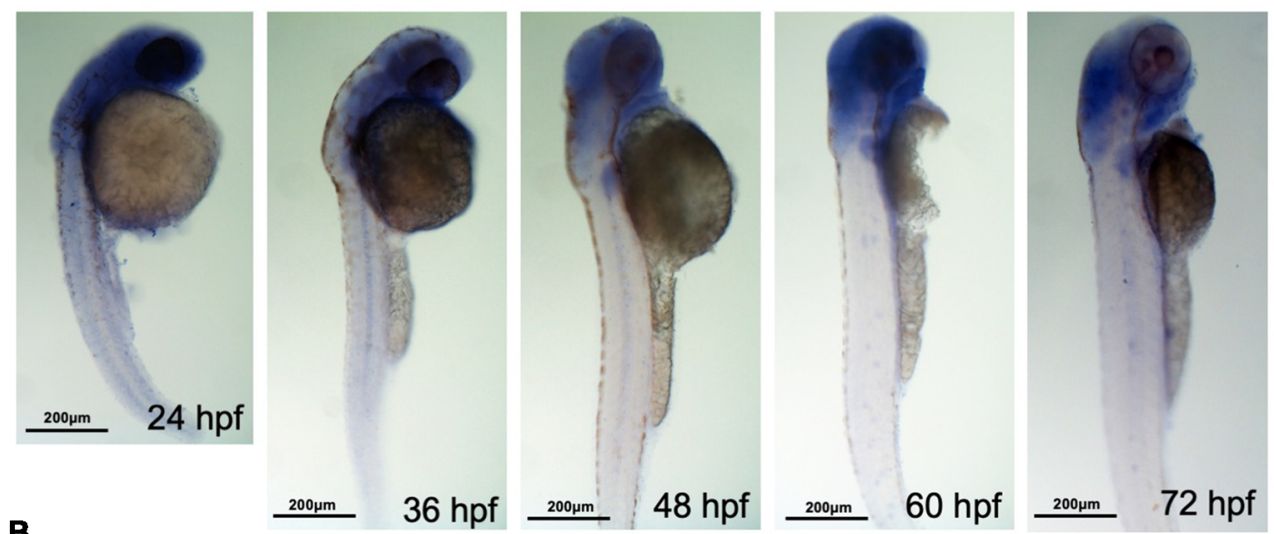

B
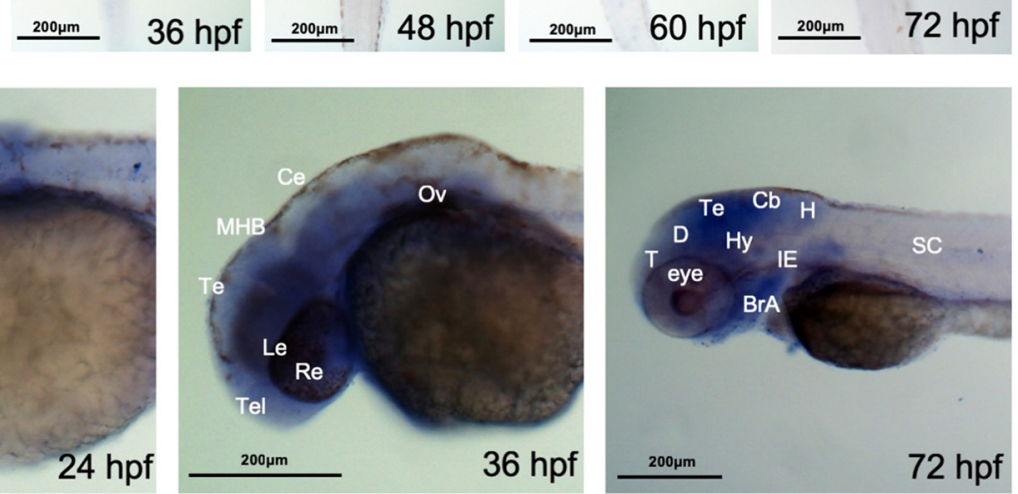

FIGURE 2 | ahi1 is highly expressed in zebrafish cephalosome. (A) Whole-mount in situ hybridization showed the spatiotemporal expression of ahi1 mRNA from 24 36, 48, 60, and $72 \mathrm{hpf}$. (B) At the very early developmental stages (24 hpf and $36 \mathrm{hpf}$ ), the rostral feature of ahi1 mRNA expression revealed that ahi1 is more highly expressed in whole brain and ocular tissue compared with 72 hpf. T, telencephalon; D, diencephalon; Te, tectum; Hy, hypothalamus; Cb, cerebellum; H, hindbrain; BrA, brachial arches; IE, inner ear; SC, spinal cord.

has been used to study how axon pathways develop and cross the midline (Hutson and Chien, 2002; Doering et al., 2008; Xu et al., 2010). The enrichment of ahil in the eye of early developing fish allowed us to examine whether altering ahil expression could affect the RGC axonal projection and eye morphology. To do so, we first knocked down ahil in zebrafish using a morpholino (MO) to target the junction of intron 12 and exon 13 (I12E13) of the fish ahil gene (Simms et al., 2012), which encodes the second WD40 domain in the middle region of fish ahil (Figure 3A). Zebrafish embryos at the 1-cell stage were injected with the ahi1 MO, and the injected embryos were collected for analysis after 3 dpf. Because of a lack of an antibody to label fish ahil, we used RT-PCR to detect the expression of ahil. In the ahil MO-injected embryos, RT-PCR with primers to amplify the ahil gene containing exon 11 and 16 clearly demonstrated the presence of a truncated ahil product at approximately a $20 \%$ level of the total ahil (Figure 3B). DNA analysis confirmed a deletion of exons 12 and 13, which resulted in a 118 aa deletion and generated a mutant ahil form lacking the intact WD40 repeats (Figure $3 \mathrm{C}$ ).

We then examined the RGC axon projection and eye morphology in ahi1 MO-injected zebrafish at $4 \mathrm{dpf}$. The fish eye tissues were fixed and then injected with dye (DiI and DiD) unilaterally to label one side of projected axons and eye tissues, respectively. In this way, the merged images would clearly reveal axonal decussation and eye size. The control eyes of zebrafishes showed symmetric and intact axonal decussation. In the ahil MO-injected fish eyes, failure of the RGC axons to exit the retina was observed frequently. For those eyes with the RGC axon projection, there were reduced or ipsilateral axonal projections, as well as decreased eye sizes (Figure 4A). Quantification of the lengths of nerve projection and $59.25 \%$ of fish with abnormal middle crossing verified that ahi1 MO can disrupt the RGC axon projection (Figure 4B). Furthermore, the area of eyes is also significantly reduced in the ahil MO-injected embryos compared with the control MO group (Figure 4C). The abnormal RGC axon projection was also verified by the ratios of different patterns of axon projection in WT (normal 98.0\%; middle crossing $0 \%$, reduced elongation 2.0\%), control MO- (normal $94.5 \%$; middle crossing $1 \%$, reduced elongation $4.5 \%$ ), ahi1 MO- (normal 41\%; middle crossing 14\%, reduced elongation $45.0 \%$ ) injected embryos (Figure 4D) and the two-dimensional scatter diagram for WT, control MO-, or ahil MO-injected embryos (Figure 4E).

ahi1 KO fish models are known to cause ciliopathy and abnormal early development (Simms et al., 2012; Elsayed et al., 2015; Lessieur et al., 2017). However, the potential RGC phenotype in the ahil KO models has yet to be 


\section{A}

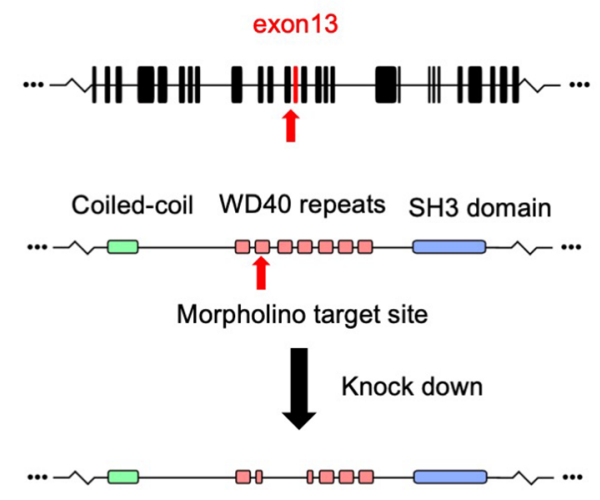

C

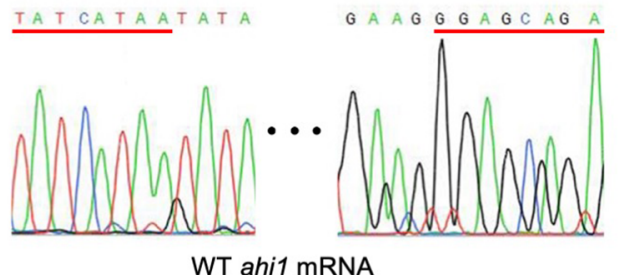

AT GCTTTTCCTATCATAAGGAGCAGAAT GT

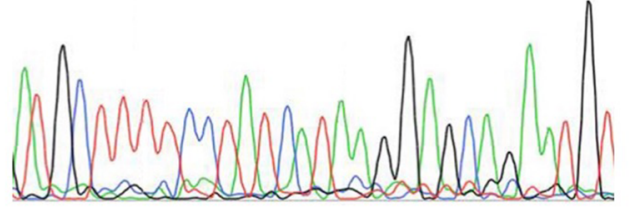

Truncated ahi1 mRNA
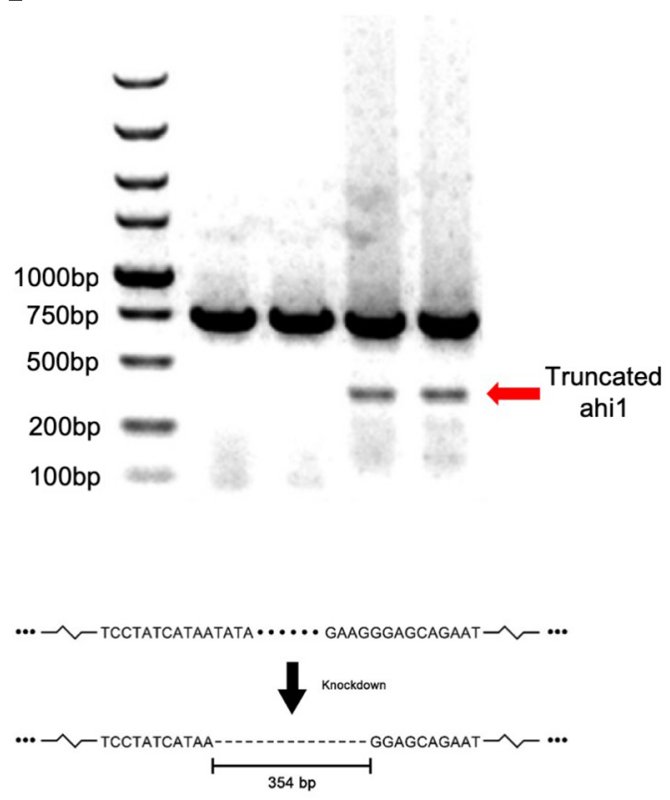

ahi1 DNA (NM_001319989)

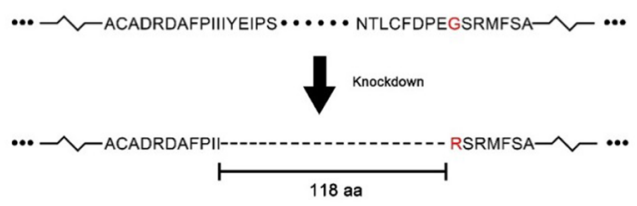

Ahi1 protein (ENSDART00000148403.2)

FIGURE 3 | Targeting the zebrafish ahi1 gene using morpholino. (A) The morpholino was designed to target the junction of intron 12 and exon 13 (112E13) of ahi1, which encodes the second WD40 repeat region of the ahi1 protein. The morpholino targeting leads to a deletion 354-bp loss of ahi1 mRNA, covering almost the whole of exon 12 to exon 13, and results in generation of a mutant ahi1 protein. (B) Mutant ahi1 mRNA was detected by RT-PCR in ahi1 morpholino-injected zebrafish. (C) The sequencing analysis of truncated ahi1 PCR product showed a deletion (354 bp) by morpholino, resulting in the generation of a mutant ahi1 with a 118 amino acid deletion.

investigated. We next examined the RGC axon projection and eye morphology in ahil KO fish. The ahil null (ahillri ${ }^{46-/-}$ ) fish model was established by targeting exon 5 of the fish ahi1 via TALENs, which results in a complete loss of the WD40 repeats and $\mathrm{SH} 3$ domain of the protein (Lessieur et al., 2017; Figure 5A). Using embryos at $4 \mathrm{dpf}$, we stained the RGC projection and eyes and found that both ahillri ${ }^{46-/-}$ and WT embryos showed the same eye sizes and indistinguishable retinotectal projection structure (Figure 5B). We did not find any abnormal middle crossing in ahillri ${ }^{46-/-}$ fish. Quantitative results also revealed no significant differences in the length and integrity of the RGC axons and size of eyes between these two groups (Figures 5C,D).

\section{CRISPR/Cas9-Mediated Ahi1 Truncation Affects Axonal Projection}

The lack of a defective eye phenotype in ahillri ${ }^{46-/-}$ fish indicates that this phenotype may be caused by a toxic gain of function of mutant ahil, rather than the complete loss of ahil. Given that morpholino can generate truncated mRNA (König et al., 2007; Morcos, 2007), it is possible that mutant ahi1 lacking the intact WD40 repeats generated by morpholino may affect axonal projection in a manner similar to defective axonal decussation in patients caused by AHI1 mutations that yield truncated AHI1. To validate this idea, we further used CRISPR/Cas9 to generate truncated ahil in zebrafish. We designed two gRNAs to target exon 11 and exon 14 in the fish ahil gene, which can create mutations to truncate ahil protein in the middle ahil region, resulting in truncated ahi1 lacking the intact WD40 repeats and the SH3 domain (Figure 6A). The zebrafish embryos were injected with these gRNAs and Cas9 protein at the one-cell stage. The injected embryos were then collected at $4 \mathrm{dpf}$ after injection for analysis. Because mosaic mutations are usually caused by CRISPR/Cas9, we had to screen and identify those zebrafish that did not develop normally and might contain the targeted ahil gene 

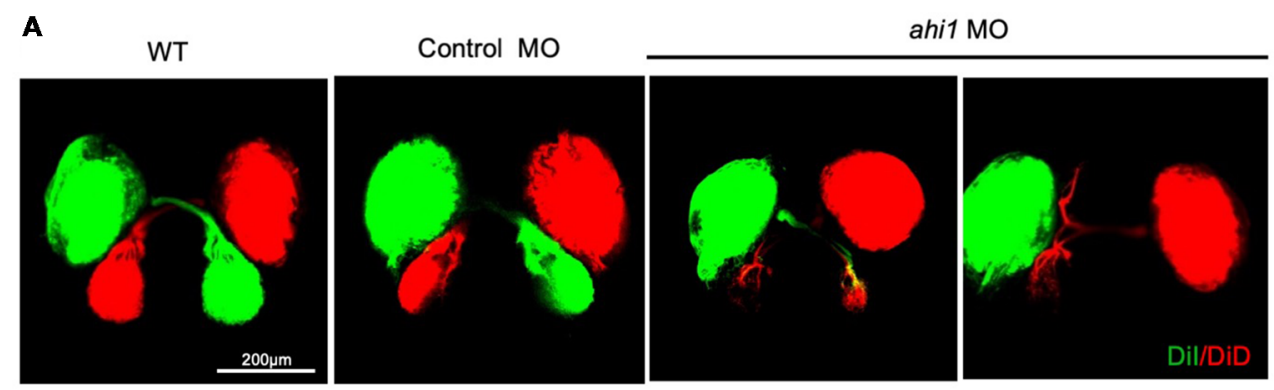

B

Length of RGC axons

Abnormal crossing (\%)

C
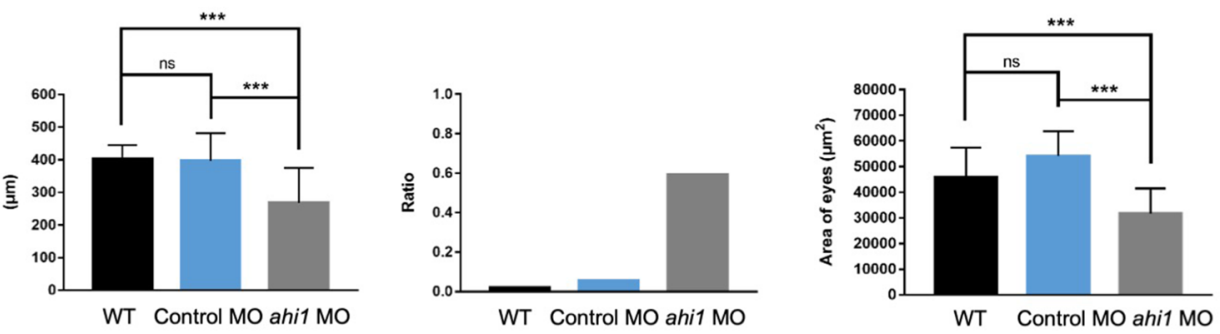

D
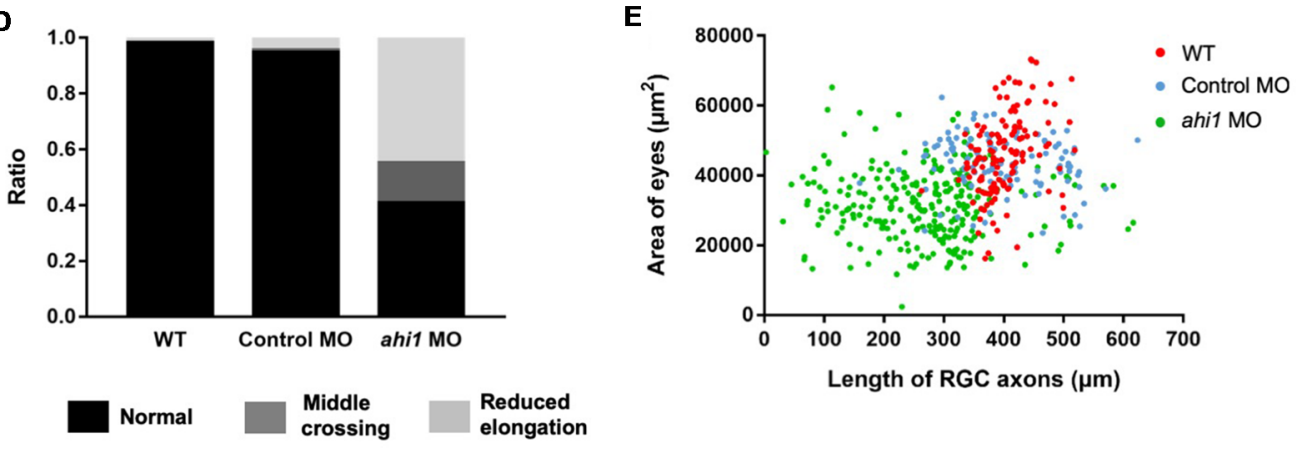

FIGURE 4 | The ahi1 morpholino-injected embryos exhibited retinal ganglion cell (RGC) axon projection defects at 4 dpf. (A) The normal retinotectal projection pattern of WT or control morpholino (Control MO)-injected embryos and defective retinotectal projections in the ahi1 morpholino (ahi1 $\mathrm{MO}$ )-injected embryos. Scale bar: $200 \mu \mathrm{m}$. (B) The statistical results of the RGC axon length and abnormal middle crossing of WT $(n=106)$, Control MO- $(n=112)$, and ahi1 MO- $(n=266)$ injected embryos. (C) The statistical results of eye sizes of WT and ahi1 MO-injected embryos. (D) The ratios of embryos with normal retinal axon projection (Normal), abnormal middle crossing (Middle crossing), and reduced axon longation (Reduced elongation) in the WT, control MO-, and ahi1 MO-injected embryos. (E) The two-dimensional scatter diagram for eye size in each group. The X-axis represents the length of OT ( $\mu \mathrm{m}^{2}$ ), and the Y-axis represents the size of eyes ( $\mu \mathrm{m}^{2}$ ). Error bars denote SEM. ${ }^{* * *} p<0.0001$ was determined via Student's $t$-test.

in their ocular tissues. Indeed, in the fish showing abnormal development, T7E1 assays of their eye tissues verified that CRISPR/Cas9 targeting ahi1 had multiple ahi1 DNA fragments and reduced levels of intact ahi1 (Figure 6B). DNA sequencing verified the indel mutations in the targeted site of the fish ahil gene, which could result in truncated ahil (Figure 6C). The CRISPR/Cas9-targeted fish showed abnormal size and curved body axis at 3 months compared to wild-type fish (Figure 6D), suggesting that ahil mutations created by CRISPR/Cas9 can affect the normal development of fish.

Next, we examined eye morphology and axonal projection in the CRISPR/Cas9-targeted fish, as we did previously. We found the same phenotypes (abnormal axonal projections and eye size reduction) in the CRISPR/Cas9-targeted fish embryos at $4 \mathrm{dpf}$ (Figure 7A). In other embryos that did not show ahil mutations, we found no such phenotypes. Quantitative results also showed that CRISPR/Cas9 targeting could yield defective axonal projection phenotypes, including reduced RGC axon projection length, the presence of abnormal crossing, and decreased eye size (Figure 7B). Taken together, using CRISPR/Cas9 to generate truncated ahi1, we also observed similar phenotypes of abnormal axonal projection in the fish ocular tissues. By measuring the ratio of different patterns of axon projection (Figure 7C) and the two-dimensional scatter diagram for WT and CRSIPR/Cas9mediated ahi1 knockout (ahi1 KO) embryos (Figure 7D), we also confirmed abnormal RGC axon projections after targeting the ahil gene by CRISPR/Cas9.

\section{DISCUSSION}

Most AHI1 mutations in JBTS patients result in truncated proteins lacking the intact WD40 repeats and the SH3 domain. 
A

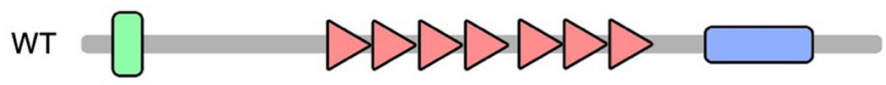

ahi1/ri46-\%

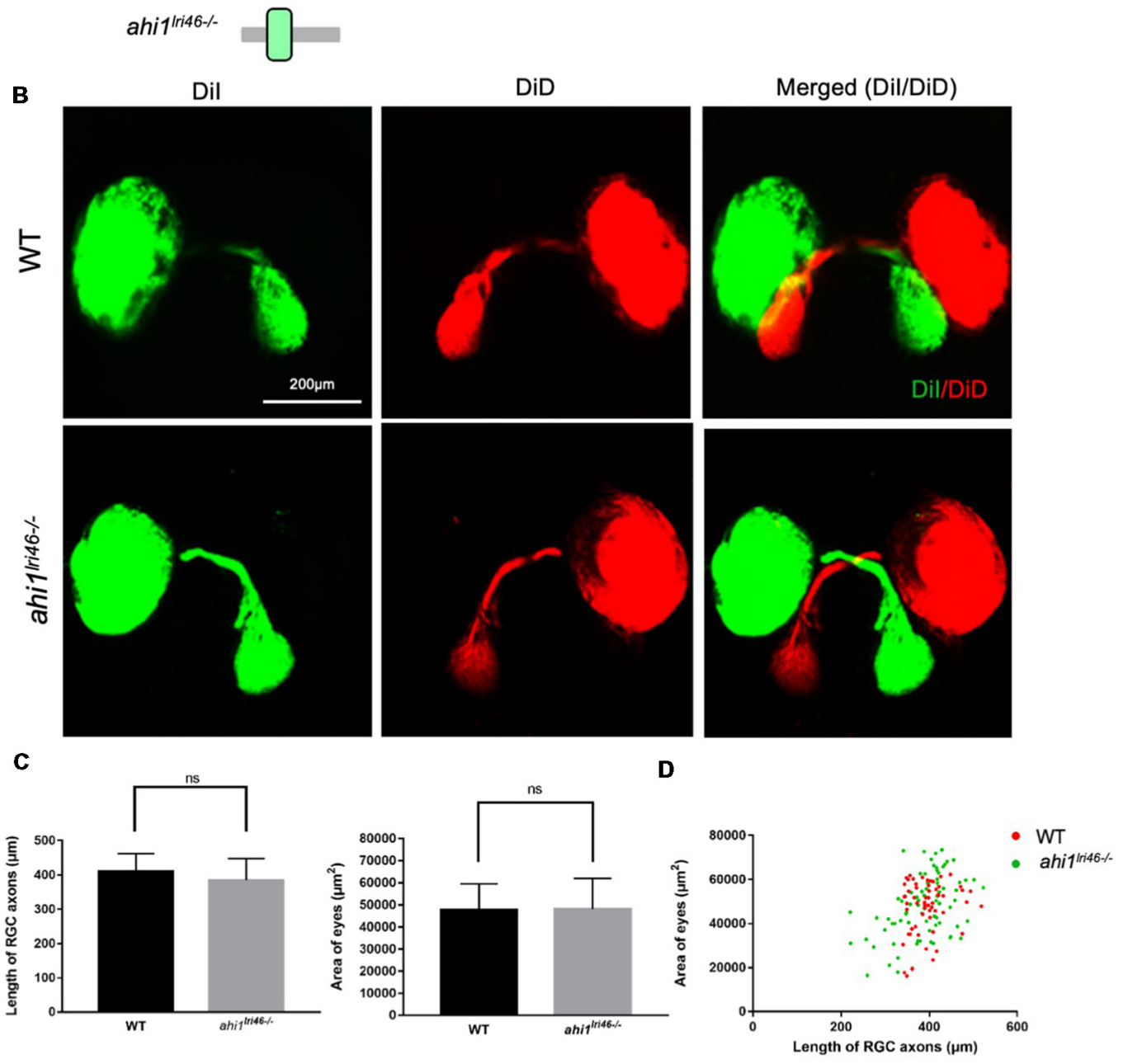

FIGURE 5 | The ahi1 knockout (KO) line ahi1/rit6-/- embryos exhibited no significant difference from WT in optic nerve projection length and eyes size. (A) The ahi1 /rit6-/- zebrafish has depleted most of the region of the ahi1 gene and only retains the N-terminal coiled-coil domain. (B) Fluorescent images of WT and ahi1/rit6-/- zebrafish optic nerve projections at 4 dpf. Scale bar: $200 \mu \mathrm{m}$. (C) The quantified optic nerve projection length and eye sizes of WT and ahi1/ri46-/embryos. (D) The two-dimensional scatter diagram for the eye size of WT and ahi1/ri ${ }^{46-/-}$ embryos; the X-axis represents the length of optic nerve projection ( $\mu \mathrm{m}^{2}$ ), and the $Y$-axis represents the size of eyes $\left(\mu \mathrm{m}^{2}\right)$. ns, not significant. Error bars denote SEM.

JBTS was therefore thought to be due to loss of AHI1 function. Using zebrafish as a model to target the fish ahil gene, however, we provided the first demonstration that mutant ahil lacking the intact WD40 repeats can disturb RGC axon projection, suggesting that AHII mutations can affect neuronal function via a toxic gain of function, although its complete loss of function may produce other phenotypes (Elsayed et al., 2015; Lessieur et al., 2017).

Our findings consist of three lines of evidence to support the above. First, morpholino targeting can cause abnormal midline crossing of the retinotectal projection. Second, CRISPR/Cas9 targeting can also replicate this phenotype. Third, the complete loss of ahil in fish failed to yield the phenotype of defective retinotectal projection, ruling out the involvement of loss of function for the defective axonal projection. Because the retinotectal projection represents axonal formation and projection during very early brain development in zebrafish (Stuermer, 1988; Trowe et al., 1996; Picker et al., 1999), the results from our study support the notion that ahil is critical for neuronal differentiation and growth during the early development stage in zebrafish and are also consistent with earlier reports that AHI1 is a susceptibility gene for schizophrenia and autism, which are also abnormal developmental brain disorders (Levi et al., 2005; Amann-Zalcenstein et al., 2006; Ingason et al., 2007, 2010; Torri et al., 2010). 


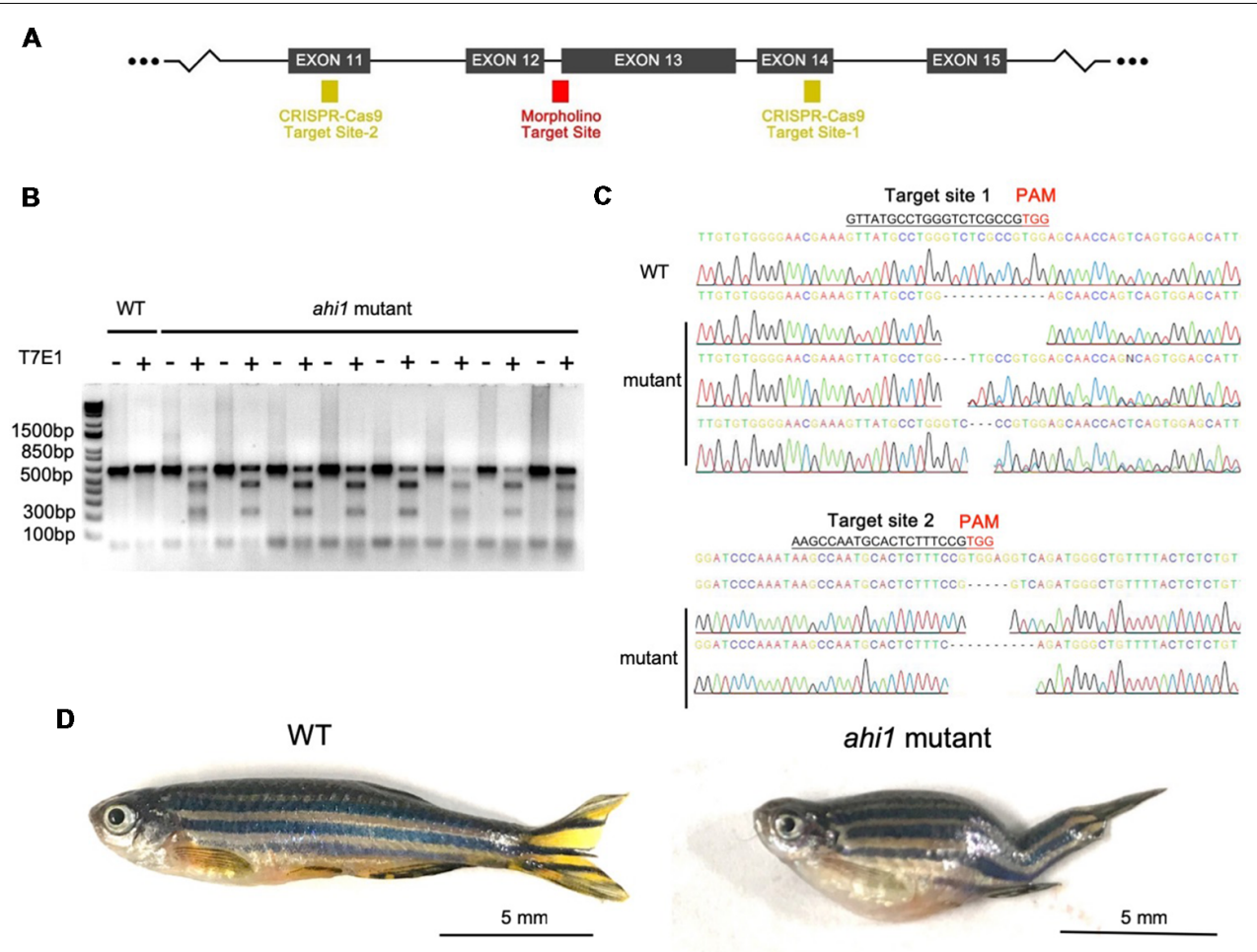

FIGURE 6 | Ahi1 mutations via CRISPR/Cas9 lead to abnormal development of zebrafish. (A) The CRISPR/Cas9 target sites were designed to create mutations in exon 12 and exon 14, which were close to the ahi1 morpholino targeting site. (B) T7E1 assay results showed cleaved ahi1 DNA products by CRISPR/Cas9. (C) Sequencing data showing ahi1 DNA mutations in the ahi1 CRSIPR/Cas9-targeted (ahi1 mutant) embryos. (D) The morphology of WT and ahi1 mutant zebrafish at 3 months.

Previous studies have established several Ahi1 KO mouse models by deleting different exons in the Ahil gene (Hsiao et al., 2009; Louie et al., 2010; Xu et al., 2010). Although these mice may also generate truncated Ahi1, they did not display axonal decussation abnormalities. One possible explanation is that the mouse Ahi1 N-terminal region lacks a coiled-coil domain, which can be a binding site to interact with many other proteins or can facilitate the interactions of other binding domains with partners (Grigoryan and Keating, 2008). Human and zebrafish Ahil do contain this coiled-coil motif, which may facilitate the abnormal interactions of the truncated Ahil with other proteins to cause a gain of function, even when truncated Ahil is expressed at the endogenous level. It seems that zebrafish eye is very sensitive to the toxicity of mutant Ahil without the intact WD40 repeats, because the abnormal retinal axon projection occurs when full-length Ahi1 is not completely depleted and when about $20 \%$ of the ahil gene was targeted by morpholino to generate mutant ahi1 fish. In humans, although JBST is a recessively inherited developmental brain disorder, heterozygous AHI1 mutations were associated with neurological symptoms (Tory et al., 2007; Otto et al., 2011), supporting a gain of function of AHI1 mutations. In mouse neurons, axonal projection can be affected by a high dose of truncated Ahil because overexpressing mouse Ahil N-terminal fragments in neuronal cells can also suppress neurite differentiation (Sheng et al., 2008; Weng et al., 2013). The species-dependent sensitivities to mutant AHI1 toxicity could be due to differences in AHI1 sequences, binding partners, and axonal structures in different species.

The toxicity of mutant ahil in zebrafish is also supported by previous findings. Truncated fish ahil lacking the intact WD40 domains, but not truncated fish ahil containing the intact WD40 and SH3 domains, could affect zebrafish development (Elsayed et al., 2015). Thus, a toxic gain of function of AHI1 is likely to be protein context-dependent. Our findings suggest that truncated ahi1 lacking the intact WD40 and SH3 domains can affect axonal projections in the fish eye tissues.

The highly stereotyped retinotectal pathway in zebrafish is determined by complex mechanisms that underlie axonal outgrowth and pathfinding. Axonal outgrowth relies critically on the active intracellular transport that supplies mitochondria, proteins, and other molecules to nerve terminals. Mouse Ahi1 is found to be involved in intracellular trafficking (Sheng et al., 2008; Westfall et al., 2010; Xu et al., 2010), which is consistent with the function of the WD40 and SSH3 domains (Huang et al., 2012; Jain, 2018) and is critical for axonal integrity and projection. Full-length Ahil is known to form a stable complex with huntingtin associated protein-1 (HAP1), which is also involved in intracellular trafficking (Sheng et al., 2008; Xiang et al., 2014). Although N-terminal Ahil does not bind Hap1 (Weng et al., 2013), an Ahil missense mutation was found to inhibit its interaction with Hap1 (Tuz et al., 2013). 

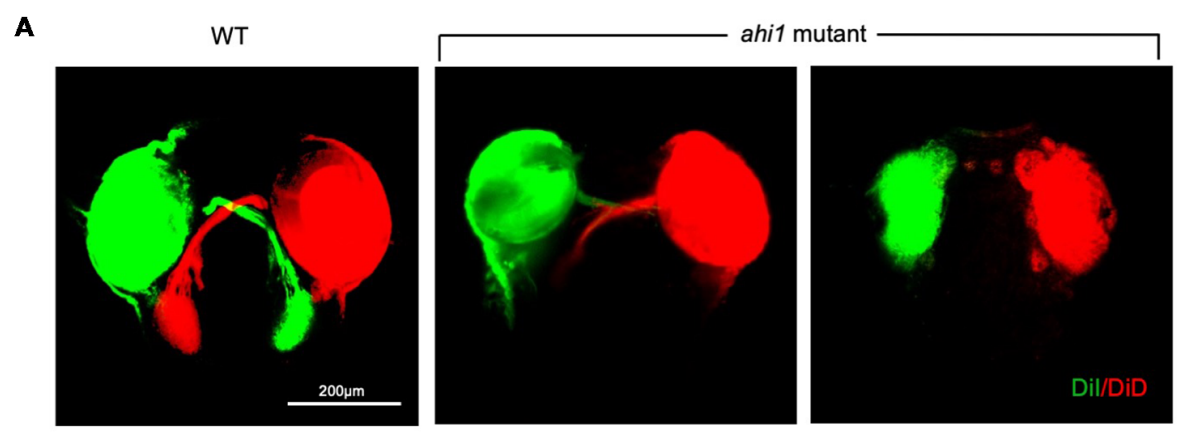

B
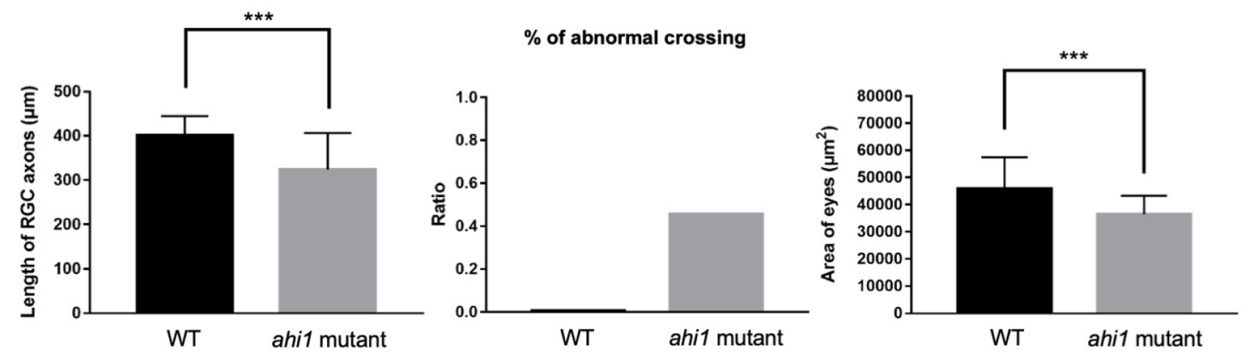

C

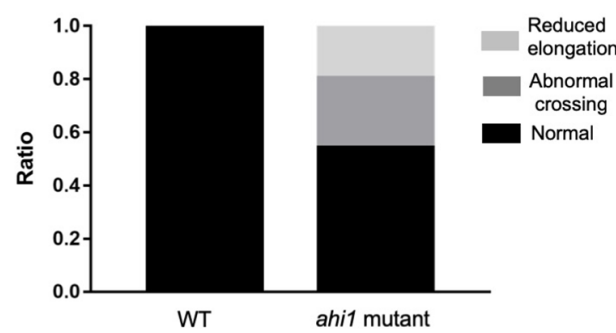

D

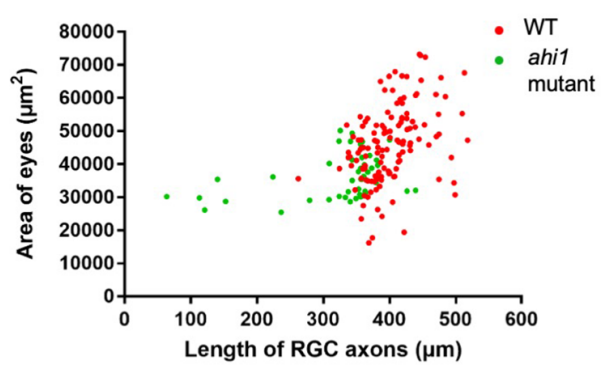

FIGURE 7 | Ahi1 CRSIPR/Cas9-targeted embryos exhibited abnormal RGC axon projection patterns compared to WT embryos. (A) Fluorescent images of the optic nerve projection of WT and ahi1 CRSIPR/Cas9 targeted (ahi1 mutant)-embryos at 4 dpf. Scale bar $=200 \mu \mathrm{m}$. (B) The statistical results of RGC axon length, abnormal crossing, and eye size of WT $(n=59)$ and ahi1 mutant $(n=46)$ embryos. (C) The ratios of embryos with normal retinal axon projection, abnormal middle crossing, and reduced axon elongation in WT and ahi1 mutant embryos. (D) The two-dimensional scatter diagram for the eye area of each WT or ahi1 mutant embryos. The X-axis represents the length of OT $\left(\mu \mathrm{m}^{2}\right)$, and the Y-axis represents the size of eyes $\left(\mu \mathrm{m}^{2}\right)$. Error bars denote SEM. ${ }^{* * *} p<0.0001$.

Also, the toxicity of N-terminal Ahil can be attenuated by overexpressed Cend1/BM88, a neuronal protein that mediates neuronal differentiation (Weng et al., 2013). Overexpressed Cend1/BM88 may bind toxic truncated Ahil to prevent its abnormal interactions with other proteins and its toxic effects on neuronal differentiation. In addition, truncated Ahil could also affect cilia function to impact axonal projection. In combination with these previous discoveries, our current findings from the zebrafish model support the idea for a toxic gain of function of mutant Ahi1, opening up a new avenue to investigate the pathogenesis of diseases associated with AHI1 mutations.

\section{AUTHOR CONTRIBUTIONS}

HX, SL, and X-JL conceived and designed the experiments. LZ, LC, and LY performed the experiments and analyzed the data. BP provided ahil $\mathrm{KO}$ fish embryos. BL provided advice. LZ and X-JL wrote the manuscript. and SL and HX edited the manuscript.

\section{FUNDING}

This work was supported by the National Natural Science Foundation of China (Grant Nos., 81160144, 81760216, 31771182 and 31171044), Young Scientist of Jiangxi Province, China (Grant No. 20122BCB23007) and NIH grant NS036232.

\section{ACKNOWLEDGMENTS}

We wish to thank Dr. Jinhu Wang and Jisheng Sun in Department of Medicine at Emory University School of Medicine for providing technical assistance and fish facility. 


\section{REFERENCES}

Amann-Zalcenstein, D., Avidan, N., Kanyas, K., Ebstein, R. P., Kohn, Y., Hamdan, A., et al. (2006). AHI1, a pivotal neurodevelopmental gene and C6orf217 are associated with susceptibility to schizophrenia. Eur. J. Hum. Genet. 14, 1111-1119. doi: 10.1038/sj.ejhg.5201675

Dixon-Salazar, T., Silhavy, J. L., Marsh, S. E., Louie, C. M., Scott, L. C., Gururaj, A., et al. (2004). Mutations in the AHI1 gene, encoding jouberin, cause Joubert syndrome with cortical polymicrogyria. Am. J. Hum. Genet. 75, 979-987. doi: $10.1086 / 425985$

Doering, J. E., Kane, K., Hsiao, Y. C., Yao, C., Shi, B., Slowik, A. D., et al. (2008). Species differences in the expression of Ahil, a protein implicated in the neurodevelopmental disorder Joubert syndrome, with preferential accumulation to stigmoid bodies. J. Comp. Neurol. 511, 238-256. doi: $10.1002 / \mathrm{cne} .21824$

Elsayed, S. M., Phillips, J. B., Heller, R., Thoenes, M., Elsobky, E., Nürnberg, G., et al. (2015). Non-manifesting AHI1 truncations indicate localized loss-offunction tolerance in a severe Mendelian disease gene. Hum. Mol. Genet. 24, 2594-2603. doi: 10.1093/hmg/ddv022

Ferland, R. J., Eyaid, W., Collura, R. V., Tully, L. D., Hill, R. S., Al-Nouri, D., et al. (2004). Abnormal cerebellar development and axonal decussation due to mutations in AHI1 in Joubert syndrome. Nat. Genet. 36, 1008-1013. doi: $10.1038 / \mathrm{ng} 1419$

Friede, R. L., and Boltshauser, E. (1978). Uncommon syndromes of cerebellar vermis aplasia. I: Joubert syndrome. Dev. Med. Child Neurol. 20, 758-763. doi: 10.1111/j.1469-8749.1978.tb15307.x

Grigoryan, G., and Keating, A. E. (2008). Structural specificity in coiled-coil interactions. Curr. Opin. Struct. Biol. 18, 477-483. doi: 10.1016/j.sbi.2008. 04.008

Hsiao, Y. C., Tong, Z. J., Westfall, J. E., Ault, J. G., Page-McCaw, P. S., and Ferland, R. J. (2009). Ahil, whose human ortholog is mutated in Joubert syndrome, is required for Rab8a localization, ciliogenesis and vesicle trafficking. Hum. Mol. Genet. 18, 3926-3941. doi: 10.1093/hmg/ ddp335

Huang, R., Fang, P., and Kay, B. K. (2012). Isolation of monobodies that bind specifically to the SH3 domain of the Fyn tyrosine protein kinase. N. Biotechnol. 29, 526-533. doi: 10.1016/j.nbt.2011.11.015

Hutson, L. D., and Chien, C.-B. (2002). Pathfinding and error correction by retinal axons: the role of astray/robo2. Neuron 33, 205-217. doi: 10.1016/S08966273(01)00579-7

Ingason, A., Giegling, I., Cichon, S., Hansen, T., Rasmussen, H. B., Nielsen, J., et al. (2010). A large replication study and meta-analysis in European samples provides further support for association of AHI1 markers with schizophrenia. Hum. Mol. Genet. 19, 1379-1386. doi: 10.1093/hmg/ddq009

Ingason, A., Sigmundsson, T., Steinberg, S., Sigurdsson, E., Haraldsson, M., Magnusdottir, B. B., et al. (2007). Support for involvement of the AHI1 locus in schizophrenia. Eur. J. Hum. Genet. 15, 988-991. doi: 10.1038/sj.ejhg. 5201848

Jain, S. (2018). Computational Models For Domain-Peptide Mediated ProteinProtein Interactions (Doctoral Dissertation). Toronto: Department of Computer Science.

Jiang, X., Hanna, Z., Kaouass, M., Girard, L., and Jolicoeur, P. (2002). Ahi-1, a novel gene encoding a modular protein with WD40-repeat and SH3 domains, is targeted by the Ahi-1 and Mis-2 provirus integrations. J. Virol. 76, 9046-9059. doi: 10.1128/jvi.76.18.9046-9059.2002

Joubert, M., Eisenring, J.-J., Preston, J., and Andermann, F. (1969). Familial agenesis of the cerebellar vermis: a syndrome of episodic hyperpnea, abnormal eye movements, ataxia, and retardation. Neurology 19, 813-813. doi: $10.1212 /$ wnl.19.9.813

König, H., Matter, N., Bader, R., Thiele, W., and Müller, F. J. C. (2007). Splicing segregation: the minor spliceosome acts outside the nucleus and controls cell proliferation. Cell 131, 718-729. doi: 10.1016/j.cell.2007.09.043

Lancaster, M. A., Gopal, D. J., Kim, J., Saleem, S. N., Silhavy, J. L., Louie, C. M., et al. (2011). Defective Wnt-dependent cerebellar midline fusion in a mouse model of Joubert syndrome. Nat. Med. 17, 726-731. doi: 10.1038/ nm. 2380

Lessieur, E. M., Fogerty, J., Gaivin, R. J., Song, P., and Perkins, B. D. (2017). The ciliopathy gene ahil is required for zebrafish cone photoreceptor outer segment morphogenesis and survival. Invest. Ophthalmol. Vis. Sci. 58, 448-460. doi: $10.1167 /$ iovs.16-20326

Levi, A., Kohn, Y., Kanyas, K., Amann, D., Pae, C.-U., Hamdan, A., et al. (2005) Fine mapping of a schizophrenia susceptibility locus at chromosome 6q23: increased evidence for linkage and reduced linkage interval. Eur. J. Hum. Genet. 13, 763-771. doi: 10.1038/sj.ejhg.5201406

Louie, C. M., Caridi, G., Lopes, V. S., Brancati, F., Kispert, A., Lancaster, M. A., et al. (2010). AHI1 is required for photoreceptor outer segment development and is a modifier for retinal degeneration in nephronophthisis. Nat. Genet. 42, 175-180. doi: 10.1038/ng.519

Louie, C. M., and Gleeson, J. G. (2005). Genetic basis of Joubert syndrome and related disorders of cerebellar development. Hum. Mol. Genet. 14, R235-R242. doi: $10.1093 / \mathrm{hmg} / \mathrm{ddi} 264$

Morcos, P. A. (2007). Achieving targeted and quantifiable alteration of mRNA splicing with Morpholino oligos. Biochem. Biophys. Res. Commun. 358, 521-527. doi: 10.1016/j.bbrc.2007.04.172

Otto, E. A., Ramaswami, G., Janssen, S., Chaki, M., Allen, S. J., Zhou, W., et al. (2011). Mutation analysis of 18 nephronophthisis associated ciliopathy disease genes using a DNA pooling and next generation sequencing strategy. J. Med. Genet. 48, 105-116. doi: 10.1136/jmg.2010.082552

Picker, A., Brennan, C., Reifers, F., Clarke, J., Holder, N., and Brand, M. (1999). Requirement for the zebrafish mid-hindbrain boundary in midbrain polarisation, mapping and confinement of the retinotectal projection. Development 126, 2967-2978.

Poirier, Y., Kozak, C., and Jolicoeur, P. (1988). Identification of a common helper provirus integration site in abelson murine leukemia virus-induced lymphoma DNA. J. Virol. 62, 3985-3992.

Sheng, G., Xu, X., Lin, Y. F., Wang, C. E., Rong, J., Cheng, D., et al. (2008). Huntingtin-associated protein 1 interacts with Ahil to regulate cerebellar and brainstem development in mice. J. Clin. Invest. 118, 2785-2795. doi: $10.1172 /$ jci35339

Simms, R. J., Hynes, A. M., Eley, L., Inglis, D., Chaudhry, B., Dawe, H. R., et al. (2012). Modelling a ciliopathy: Ahi1 knockdown in model systems reveals an essential role in brain, retinal, and renal development. Cell. Mol. Life Sci. 69, 993-1009. doi: 10.1007/s00018-011-0826-Z

Stuermer, C. A. (1988). Retinotopic organization of the developing retinotectal projection in the zebrafish embryo. J. Neurosci. 8, 4513-4530. doi: 10.1523/JNEUROSCI.08-12-04513.1988

Thisse, C., and Thisse, B. (2008). High-resolution in situ hybridization to whole-mount zebrafish embryos. Nat. Protoc. 3, 59-69. doi: 10.1038/nprot. 2007.514

Torri, F., Akelai, A., Lupoli, S., Sironi, M., Amann-Zalcenstein, D., Fumagalli, M., et al. (2010). Fine mapping of AHI1 as a schizophrenia susceptibility gene: from association to evolutionary evidence. FASEB J. 24, 3066-3082. doi: 10.1096/fj. 09-152611

Tory, K., Lacoste, T., Burglen, L., Morinière, V., Boddaert, N., Macher, M. A., et al. (2007). High NPHP1 and NPHP6 mutation rate in patients with Joubert syndrome and nephronophthisis: potential epistatic effect of NPHP6 and AHI1 mutations in patients with NPHP1 mutations. J. Am. Soc. Nephrol. 18, 1566-1575. doi: 10.1681/asn.2006101164

Trowe, T., Klostermann, S., Baier, H., Granato, M., Crawford, A. D., Grunewald, B., et al. (1996). Mutations disrupting the ordering and topographic mapping of axons in the retinotectal projection of the zebrafish, Danio rerio. Development 123, 439-450.

Tuz, K., Hsiao, Y. C., Juarez, O., Shi, B., Harmon, E. Y., Phelps, I. G., et al. (2013). The Joubert syndrome-associated missense mutation (V443D) in the Abelsonhelper integration site 1 (AHI1) protein alters its localization and proteinprotein interactions. J. Biol. Chem. 288, 13676-13694. doi: 10.1074/jbc.M112. 420786

Weng, L., Lin, Y. F., Li, A. L., Wang, C. E., Yan, S., Sun, M., et al. (2013). Loss of Ahil affects early development by impairing BM88/Cend1-mediated neuronal differentiation. J. Neurosci. 33, 8172-8184. doi: 10.1523/JNEUROSCI.0119 $-13.2013$

Westfall, J. E., Hoyt, C., Liu, Q., Hsiao, Y. C., Pierce, E. A., Page-McCaw, P. S., et al. (2010). Retinal degeneration and failure of photoreceptor outer segment formation in mice with targeted deletion of the Joubert syndrome gene, Ahil. J. Neurosci. 30, 8759-8768. doi: 10.1523/JNEUROSCI.522909.2010 
Xiang, J., Yang, H., Zhao, T., Sun, M., Xu, X., Zhou, X.-F., et al. (2014). Huntingtin-associated protein 1 regulates postnatal neurogenesis and neurotrophin receptor sorting. J. Clin. Invest. 124, 85-98. doi: 10.1172/ jci69206

Xu, X., Leinwand, S. G., Dell, A. L., Fried-Cassorla, E., and Raper, J. A. (2010). The calmodulin-stimulated adenylate cyclase ADCY8 sets the sensitivity of zebrafish retinal axons to midline repellents and is required for normal midline crossing. J. Neurosci. 30, 7423-7433. doi: 10.1523/JNEUROSCI.0699-10.2010

Yachnis, A. T., and Rorke, L. B. (1999). Neuropathology of Joubert syndrome. J. Child Neurol. 14, 655-659. doi: 10.1177/088307389901 401006
Conflict of Interest Statement: The authors declare that the research was conducted in the absence of any commercial or financial relationships that could be construed as a potential conflict of interest.

Copyright (C) $2019 \mathrm{Zhu}$, Chen, Yan, Perkins, Li, Li, Xu and Li. This is an open-access article distributed under the terms of the Creative Commons Attribution License (CC BY). The use, distribution or reproduction in other forums is permitted, provided the original author(s) and the copyright owner(s) are credited and that the original publication in this journal is cited, in accordance with accepted academic practice. No use, distribution or reproduction is permitted which does not comply with these terms. 